\title{
An LC-MS/MS Method for Simultaneous Quantification of Seven Anti-HIV Medicines in Plasma of HIV-infected Patients
}

\author{
Li-jun Zhang ${ }^{1,3 *}$, Ya-min Yao ${ }^{1 *}$, Jian-jun Sun ${ }^{1}$, Jun Chen ${ }^{1}$, Xiao-fang Jia ${ }^{1}$, Fang Shen ${ }^{1}$ and Hong-zhou Lu ${ }^{1,2 *}$ \\ ${ }^{1}$ Shanghai Public Health Clinical Center, Fudan University, Shanghai 201508, China \\ ${ }^{2}$ Huashan Hospital, 12 Wu Lu Mu Qi Medium Road, Shanghai 200040, China \\ 3Institute of Clinical Pharmacology, Pharmacogenetics Research Institute, Changsha, Hunan 410078, China
}

\begin{abstract}
A quick and high-through LC-MS/MS method has been developed and applied to simultaneously quantify lamivudine (3TC), stavudine(d4T), zidovudine(AZT), efavirenz(EFV), nevirapine(NVP), and Lopinavir/Ritonavir (LPV/RTV) plasma concentrations. A combination of protein precipitation and liquid-liquid extraction was used to extract all compounds. The method showed a good linearity in a concentration range of $20-3200 \mu \mathrm{g} / \mathrm{L}$ for $3 T \mathrm{TC}, \mathrm{d} 4 \mathrm{~T}$, AZT, $40-6400 \mu \mathrm{g} / \mathrm{L}$ for EFV and NVP, $62.5-10000 \mu \mathrm{g} / \mathrm{L}$ for LPV and $12.5-2000 \mu \mathrm{g} / \mathrm{L}$ for RTV. Mean intra- and inter-day precision were within $\pm 20 \%$ at the LLOQ and $\pm 15 \%$ at the other QC level. The accuracy were between $85 \%$ and $115 \%$ for all seven analytes. The whole run is 13 minutes. This method has been successfully used for analyzing 133 samples from 84 HIV-positive patients being treated with the combination therapy in China. To our knowledge, it is the first time to use LC-MS/MS method to simultaneously quantify 3TC, d4T, AZT, EFV, NVP, LPV and RTV in China for investigating the relationship between blood drug concentrations and efficacy and/or toxicity.
\end{abstract}

Keywords: LC-MS/MS; Plasma concentration; Anti-HIV medicine; HIV-positive patient

\section{Introduction}

In recent years, highly active antiretroviral therapy (HAART) has become the standard care for the management of patients with human immunodeficiency virus (HIV) infection [1]. With the wide use of these antiretroviral medicines, the survive ratio of HIV-infected patients has greatly improved. However, some new questions come out, such as drug resistance, drug toxicity and drug-drug interactions. Several studies [2-4] have demonstrated a relationship between plasma drug concentrations and efficacy and/or toxicity. Therefore, therapeutic drug monitoring (TDM) of antiretroviral drugs is important in clinical care $[5,6]$ to determine the best dosage regimen adapted to each individual to reduce the risk of virologic failure from low plasma drug concentrations and to limit the toxicity linked to high plasma drug concentrations.

In China, the first-line antiretroviral combination includes two nucleoside reverse transcriptase inhibitors (NRTIs) and a non-nucleoside reverse transcriptase inhibitors (NNRTI) or Protease Inhibitor (PI), such as AZT+3TC+EFV/NVP/(LPV/RTV) and $\mathrm{d} 4 \mathrm{~T}+3 \mathrm{TC}+\mathrm{NVP} / \mathrm{EFV} /(\mathrm{LPV} / \mathrm{RTV})$, which are widely used in clinical practice. Since only few combination options are available in China and the dosage of these medicines is primarily based on the clinical data from Caucasians. Is it the most appropriate dosage for Chinese patients with HAART?

In order to answer the above questions, first of all, what we need to do is to build a quick and high-through method to detect these medicines. Now several bioanalytical methods, mostly employing either high performance liquid chromatography (HPLC) with UV detection [7] or HPLC tandem mass spectrometry (LC-MS/MS) [8-11] have been reported for quantifying AZT, 3TC, d4T, EFV, NVP, etc. Some of them $[8,11]$ used SPE columns, however, SPE column is expensive and not very suit for Chinese clinical use.

So in this work, we developed and validated a simple and sensitive LC-MS method for simultaneous detection of AZT, 3TC, d4T, EFV, NVP and LPV/RTV in human plasma. This method was successfully used to study the relationship between the plasma drug concentration and therapeutic efficacy in China.

\section{Material and Methods}

\section{Chemicals}

Working standard of lamivudine (3TC), stavudine (d4T), zidovudine (AZT), efavirenz (EFV), nevirapine (NVP), lopinavir (LPV), ritonavir (RTV) and telmisartan (internal standard, IS) were supplied by U.S Pharmacopeia. HPLC grade methanol and acetonitrile were purchased from Merck Ltd., Germany. Analytical reagent (AR) grade ammonium acetate and glacial acetic acid were purchased from Sinopharm chemical Ltd., China. Blank human blood was collected in the tubes containing Ethylene Diamine Tetra acetic Acid (EDTA) from healthy and drug free volunteers. After centrifugation at $4000 \mathrm{rpm}$ at room temperature, plasma was collected and stored at $-20^{\circ} \mathrm{C}$.

\section{LC-MS/MS conditions}

An HPLC system (Shimadzu, Kyoto, Japan) consisting of a binary LC-20AD prominence pump, autosampler (SIL-HTC), solvent degasser (DGU-20A3 prominence) and a temperature controlled compartment for column (CTO10AVP) were used for all the analytes. The chromatographic system consisted of Eclipse XDB-C18 $(150 \mathrm{~mm}$ $\times 4.6 \mathrm{~mm}, 5 \mu \mathrm{m})$ analytical column bought from Agilent Company (USA). The flow rate of the mobile phase was kept at $0.5 \mathrm{~mL} / \mathrm{min}$. The autosampler temperature was set at $4^{\circ} \mathrm{C}$ and the injection volume was $10 \mu \mathrm{L}$. The mobile phase consisted of $\mathrm{A}(0.1 \%$ formic acid in

*Corresponding authors: Li-jun Zhang, Shanghai Public Health Clinical Center Fudan University, Shanghai 201508, China, E-mail: zhanglijun1221@163.com

Hongzhou Lu, Shanghai Public Health Clinical Center, Fudan University, Shanghai 201508, China, E-mail: luhongzhou@fudan.edu.cn

Received August 23, 2010; Accepted September 23, 2010 Published September 25,2010

Citation: Zhang LJ, Yao YM, Sun JJ, Chen J, Jia XF, et al. (2010) An LC-MS/MS Method for Simultaneous Quantification of Seven Anti-HIV Medicines in Plasma of HIV-infected Patients. Pharm Anal Acta 1:102. doi:10.4172/2153-2435.1000102

Copyright: ( $\odot 2010$ Zhang LJ, et al. This is an open-access article distributed unde the terms of the Creative Commons Attribution License, which permits unrestricted use, distribution, and reproduction in any medium, provided the original author and source are credited. 
water): B (0.1\% formic acid in methanol) $(20: 80, \mathrm{v} / \mathrm{v})$. The column oven temperature was maintained at $30^{\circ} \mathrm{C}$. The pressure of the system was 4.5 $\mathrm{MPa}$ and the total $\mathrm{LC}$ run time was 13 minutes.

Detection of analytes and IS were performed on a triple quadrupole mass spectrometer, API-3200 (Applied Biosystems, USA) equipped with turbo ion spray ionization source and operating in the positive ion mode. Analyst software version 1.4.2 was used to control all parameters of LC and MS. Quantification was performed using multiple reaction monitoring (MRM) mode based on the precursor $\mathrm{m} / \mathrm{z}$ and its fragment $\mathrm{m} / \mathrm{z}$ (MRM transition) for each analyte. Sourcedependent parameters optimized were: gas 1 (nebulizer gas), 40 psi; gas 2 (heater gas), 50psi; ion spray voltage (ISV), 4500V; temperature (TEM), $550^{\circ} \mathrm{C}$. Compound-dependent parameters like declustering potential (DP), entrance potential (EP), collision energy (CE), cell exit potential (CXP) and dwell time were optimized. Nitrogen was used as collision activated dissociation (CAD) gas and was set at 6 psi. Quadrupoles 1 and 3 were maintained at low and unit resolution, respectively.

\section{Stock solutions}

Stock solutions of AZT, d4T, 3TC, NVP, LPV and RTV were prepared as $1.0 \mathrm{~g} / \mathrm{L}$ concentrations in $50 \%$ methanol/water and EFV prepared as $1.0 \mathrm{~g} / \mathrm{L}$ in $70 \%$ methanol/water. A $0.1 \mathrm{~g} / \mathrm{L}$ stock solution of internal standard (telmisartan, IS) in $50 \%$ methanol/water was prepared. All stock solutions were stored in $-20^{\circ} \mathrm{C}$.

\section{Standard and quality control preparation}

Each standard stock solution was diluted in $50 \%$ methanol to give a working solution containing all drugs at 200, 400, 800, 1600, 3200, $6400,12,800,25,600$ and $32,000 \mu \mathrm{g} / \mathrm{L}$ for $\mathrm{d} 4 \mathrm{~T}, 3 \mathrm{TC}$ and AZT, 400, $800,1600,3200,6400,12,800,25,600,51,200$ and $64,000 \mu \mathrm{g} / \mathrm{L}$ for EFV and NVP, 625, 800, 6250, 8000, 12,500, 25,000, 50,000, 80,000 and $100,000 \mu \mathrm{g} / \mathrm{L}$ for $\mathrm{LPV}, 125,150,1250,1500,2500,5000,10,000$, 15,000 and $20,000 \mu \mathrm{g} / \mathrm{L}$ for RTV. The internal standard was diluted in $50 \%$ methanol to give a working solution of $100 \mathrm{ng} / \mathrm{mL}$. $20 \mu \mathrm{L}$ working standard and $20 \mu \mathrm{L}$ IS was further diluted in $200 \mu \mathrm{L}$ drug free human plasma to prepare 6 plasma calibrators at 10 -fold dilution. Quality controls samples were made in blank human plasma at 40, 320 and $2560 \mu \mathrm{g} / \mathrm{L}$ for $\mathrm{d} 4 \mathrm{~T}, 3 \mathrm{TC}$ and AZT, 80, 640 and $5120 \mu \mathrm{g} / \mathrm{L}$ for EFV and NVP, 80,800 and $8000 \mu \mathrm{g} / \mathrm{L}$ for LPV and 15,150 and $1500 \mu \mathrm{g} / \mathrm{L}$ for RTV.

\section{Sample preparation}

Owing to the varying physicochemical properties of the compounds, a protein precipitation followed by a liquid-liquid extraction method was used to prepare plasma samples for the quantification of antiretroviral agents. Samples in $0.2 \mathrm{~mL}$ plasma were transferred into a $2.0 \mathrm{~mL}$ centrifuge tubes and $0.2 \mathrm{~mL}$ of organic solvent $\left(50 \mathrm{mM} \mathrm{NH}_{4} \mathrm{Ac}: \mathrm{ACN}=1: 6\right)$ were added for protein precipitation and votexed $3 \mathrm{~min}$ for sufficient mixing. Then $1.0 \mathrm{~mL}$ ethyl acetate were added to above samples to extract anlytes to organic layer and keep the endogenous materials from plasma which were mostly water soluble in aqueous layer to reduce matrix effect. The resulting samples were vortexed for 5 minutes and centrifuged at 12,000 rpm for 10 minutes at $4^{\circ} \mathrm{C}$. The supernatant was transferred to a new tube and evaporated under nitrogen at $37^{\circ} \mathrm{C}$. A second ethyl acetate extraction was performed after centrifugation and the organic layer was added to the first dried extract and evaporated as described and the residue was redissolved in $200 \mu \mathrm{L}$ mobile phase. A $10 \mu \mathrm{L}$ aliquot was injected into the HPLC-MS system.

\section{Method validation}

Specificity: Analytic interferences from endogenous substances were investigated by analyzing drug-free human plasma samples from six individuals.

Matrix effects: Ion suppression/enhancement due to matrix was checked according to the previous publications $[8,12]$. Five different batches of blank plasma were extracted as described in section 2.5 and then spiked with the analytes at high quality control (HQC), middle quality control (MQC) and low quality control (LQC). The corresponding peak areas of the analytes in spiked plasma post-extraction (A) were then compared to those of the aqueous standards in $50 \%$ methanol (B) at equivalent concentrations. The ratio $(\mathrm{A} / \mathrm{B} \times 100)$ is defined as the matrix effect $(\mathrm{ME})$. A ME value of $100 \%$ indicates that the responses for analytes in the mobile phase and in the plasma extracts were the same and that no absolute ME was observed. A value of $>100 \%$ indicates ionization enhancement and a value of $<100 \%$ indicates ionization suppression.

Limit of quantization (LOQ) and limit of detection (LOD): LOD was set to the ratio of signal/noise $(\mathrm{S} / \mathrm{N})$ bigger than 3.0 in chromatogram. LOQ was set to $S / N$ more than 10 .

Calibration curves: Calibration curves were analyzed by weighted linear regression $\left(1 / \mathrm{X}^{2}\right)$ of the area ratio of each antiretroviral drug to internal standard versus nominal drug concentrations. Each calibration standard were prepared and analyzed in five independent batches. To assess linearity, deviations of the mean calculated concentrations should be within $\pm 15 \%$ while lower limit of quantification (LLOQ) within $\pm 20 \%$ from nominal concentrations for the non-zero calibration standards. Unknown concentrations were calculated from the linear regression equation of the peak area ratio against the concentration of each antiretroviral agent.

Precision, accuracy and recovery: The precision of the method was determined by the replicate analyses of QC samples. For intraday precision, three QCs (HQC, MQC and LQC) were involved. Five independent samples were prepared for each QC and analyzed in the same standard curve. For inter-day CV, 15 independent samples were prepared and analyzed in triplicate days. The accuracy of the method was expressed by ((mean observed concentration)/(spiked concentration) $) \times 100 \%$. The absolute recovery was determined by comparing the peak area of analyte extracted from plasma to that of standards injected directly.

Analysis of clinical samples: The developed method was used to analyze 133 samples from 84 patients (69 males and 15 females) aged 18 years or older who participated in our TDM study that was approved by the Ethical Committee of Shanghai Public Health Clinical Center and with the patients' consent. According to patients' baseline, six types of antiretroviral combination were used, including AZT/3TC/NVP (34 samples, 25.6\%); AZT/3TC/EFV (50, 37.6\%); d4T/3TC/ $\operatorname{NVP}(18,13.5 \%) ;$ d4T/3TC/EFV (17, 12.8\%); AZT/3TC/LPV/RTV (12, 9.0\%) and 3TC/EFV/LPV/RTV $(2,1.5 \%)$. The dosage and times of ARV are as follows: AZT, $300 \mathrm{mg}$, twice a day; $3 \mathrm{TC}, 300 \mathrm{mg}$, once daily; $\mathrm{d} 4 \mathrm{~T}$, $30 \mathrm{mg}$, twice a day; NVP, $200 \mathrm{mg}$, twice a day; EFV, $600 \mathrm{mg}$, once daily and LPV (400mg) / RTV (100mg), twice a day. Blood samples were collected during the follow up in our clinic or hospital. Blood samples were collected into $5 \mathrm{ml}$ EDTA tubes. The drug concentrations were assayed using the LC-MS/MS procedure. Clinical samples were diluted or concentrated so that the drugs concentration obtained were within the linear range of the assay if necessary. 


\section{Results and Discussions}

\section{LC-MS of AZT, d4T, 3TC, EFV, NVP, LPV, RTV and IS and specificity of} this method

For optimum detection and simultaneous quantification of AZT, d4T, 3TC, EFV NVP, LPV and RTV with IS in human plasma, it was necessary to adjust not only the chromatographic conditions and mass parameters but also to develop an efficient extraction method that gives consistent and reproducible recovery of analyte from plasma. Precursor ions and product ions were optimized by infusing $1000 \mu \mathrm{g} / \mathrm{L}$ solutions into mass spectrometer in suitable mass range respectively, both in positive and negative polarity modes using Electrospray Ionization technique. Best intensity for $[\mathrm{M}+\mathrm{H}]^{+}$ ions was found in positive mode for all eight analytes including IS as they have an ability to accept protons. The compounds and their molecullar weight, precursor ions, product ions, retention time, were listed in Table 1.

The LC-MS/MS method has high selectivity for only a precursor $\rightarrow$ product ion of the analyte of interest monitored by MRM. The selectivity towards endogenous plasma components was assessed in six different batches of human plasma samples by analyzing blanks and spiked samples at LQC levels. Endogenous peaks at the retention time of the analytes were not observed in any of the plasma batches (Figure 1A), all analytes in the plasma spiked at the LQC level (Figure 1B) and clinical samples (Figure 1C) can be detected in their retention time with single peak. This indicated that there was no significant direct interference in the MRM channel for the analytes at the expected retention time. This method is quick and easy for all analytes can be analyzed with the total run time of 13 minutes under positive mode.

\section{Matrix effects (ME)}

The ME data at QC concentrations of analytes in five different lots of human plasma are presented in Table 2. There was some ME, as indicated by values of slightly $<100 \%$ or $>100 \%$ for plasma samples spiked post-extraction. The ME observed was similar to the QC concentration ranges, without showing any analyte concentrationdependence for different lots of human plasma. This indicated a small ionization enhancement or suppression for analytes (86.2-116.2\%) under the present chromatographic and extraction conditions when the ESI interface was employed. However, such ionization suppression did not affect the slopes and linearity of the established calibration curves for the matrix matched standards over the analytical period.

\section{LOQ and LOD}

Results for LOQ and LOD were shown in Table 3. The LOQs are much lower than the reports especially for d4T and 3TC $[8,11]$. In these two studies, LOQ was 20 to $50 \mu \mathrm{g} / \mathrm{L}$. During the clinical application, we detected a lot of samples with plasma concentration

\begin{tabular}{|l|l|l|l|l|}
\hline $\begin{array}{l}\text { Antiretroviral } \\
\text { agents }\end{array}$ & $\begin{array}{l}\text { Molecullar } \\
\text { weight }\end{array}$ & $\begin{array}{l}\text { precursor ion } \\
{[\mathrm{M}+\mathrm{H}]^{+}}\end{array}$ & $\begin{array}{l}\text { product ion } \\
{[\mathrm{M}+\mathrm{H}]^{+}}\end{array}$ & $\begin{array}{l}\text { retention } \\
\text { time } \\
\text { (minutes) }\end{array}$ \\
\hline AZT & 267 & 268.0 & 127.0 & 3.22 \\
\hline 3TC & 229 & 230.2 & 112.2 & 2.67 \\
\hline d4T & 224 & 225.0 & 126.8 & 3.00 \\
\hline NVP & 266 & 267.0 & 226.0 & 3.55 \\
\hline EFV & 315 & 316.0 & 243.9 & 6.62 \\
\hline LPV & 628 & 629.3 & 155.4 & 10.66 \\
\hline RTV & 720 & 721.6 & 268.3 & 7.68 \\
\hline IS & 513 & 514.8 & 275.9 & 3.23 \\
\hline
\end{tabular}

Table 1: The ion information of 7 analytes and IS

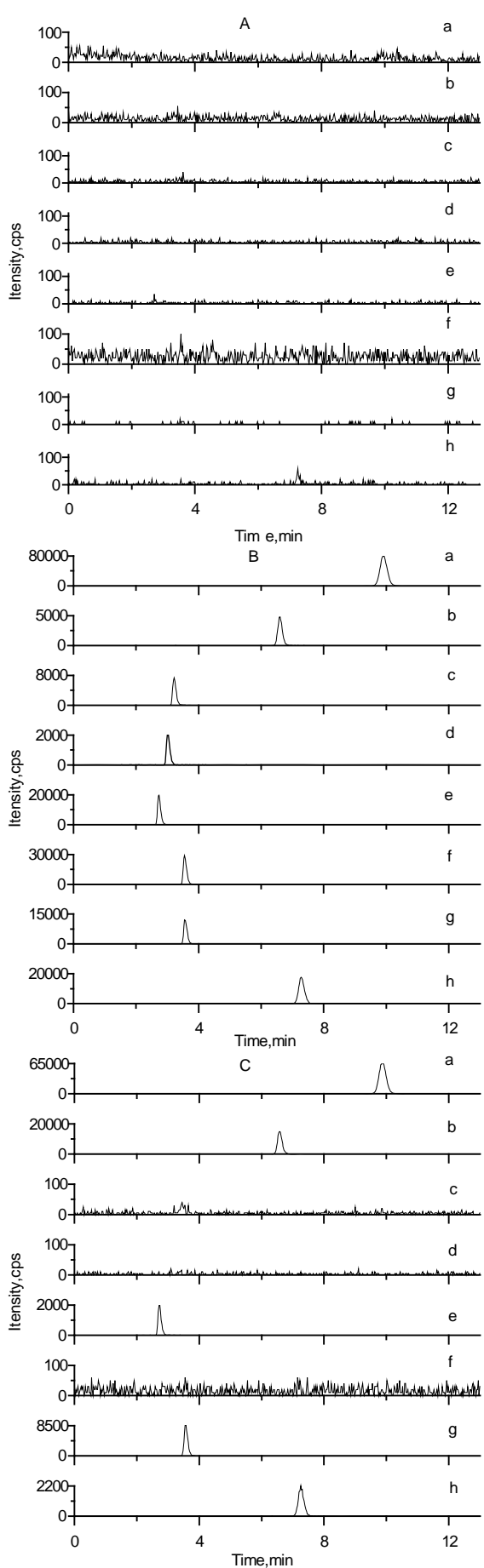

Figure 1: Specificity of method. A) MRM of blank plasma, B) MRM of spiked plasma, C) MRM of samples from patients after NVP+AZT+LPV+RTV treatment, a,b,c,d,e,f,g and $h$ represent the spectrum of IS, NVP, 3TC, d4T, AZT, EFV, LPV and RTV respectively.

lower than $20 \mu \mathrm{g} / \mathrm{L}$ for $\mathrm{d} 4 \mathrm{~T}$ and $3 \mathrm{TC}$. So it is necessary to improve the LOQ of previous method [8]. In this research, we improved the LOQs of these antiretroviral agents. This method might be much suitable for clinical research at least in China.

\section{Recovery and stability}

Five replicates at LQC, MQC and HQC level were prepared for 


\begin{tabular}{|l|l|l|l|l|l|l|l|}
\hline Analytes & NVP & AZT & D4T & 3TC & EFV & LPV & RTV \\
\hline \multicolumn{7}{|l|}{ ME(\%) } \\
\hline LQC & 96.7 & 86.2 & 91.8 & 91.3 & 97.3 & 116.2 & 92.4 \\
\hline MQC & 97.0 & 92.3 & 91.8 & 95.5 & 93.1 & 113.3 & 94.8 \\
\hline HQC & 101.3 & 95.9 & 95.3 & 99.0 & 101.2 & 104.3 & 111.3 \\
\hline
\end{tabular}

Table 2: Matrix effect data for seven analytes at $L Q C, M Q C, H Q C$ in five different lots of human plasma $(n=5)$.

\begin{tabular}{|l|l|l|}
\hline Compounds & LOD $\left(\mu \mathrm{g} \cdot \mathrm{L}^{-1}\right)$ & $\mathrm{LOQ}\left(\mu \mathrm{g} \cdot \mathrm{L}^{-1}\right)$ \\
\hline AZT & 0.25 & 1 \\
\hline 3TC & 0.5 & 2 \\
\hline $\mathrm{d} 4 \mathrm{~T}$ & 1.0 & 3 \\
\hline EFV & 1.0 & 2 \\
\hline NVP & 0.2 & 0.5 \\
\hline LPV & 0.015 & 0.05 \\
\hline RTV & 0.008 & 0.015 \\
\hline
\end{tabular}

Table 3: Limits of detection (LOD) and Limits of quantitation (LOQ) of AZT, 3TC, d4T, EFV, NVP, LPV and RTV in human plasma(n=3).

\begin{tabular}{|c|c|c|c|}
\hline Analytes & $\begin{array}{l}\text { Concentration } \\
\left(\mu \mathrm{g} \cdot \mathrm{L}^{-1}\right)\end{array}$ & Recovery (\%) & Precision (\%) \\
\hline \multirow[t]{3}{*}{ AZT } & 40 & 82.4 & 6.6 \\
\hline & 320 & 88.5 & 6.7 \\
\hline & 2560 & 97.2 & 6.8 \\
\hline \multirow[t]{3}{*}{ 3TC } & 40 & 59.3 & 8.7 \\
\hline & 320 & 53.3 & 9.3 \\
\hline & 2560 & 57.1 & 8.3 \\
\hline \multirow[t]{3}{*}{$\mathrm{d} 4 \mathrm{~T}$} & 40 & 67.6 & 12.3 \\
\hline & 320 & 70.3 & 12.9 \\
\hline & 2560 & 73.3 & 9.5 \\
\hline \multirow[t]{3}{*}{ NVP } & 80 & 105.4 & 10.4 \\
\hline & 640 & 99.8 & 8.9 \\
\hline & 5120 & 101.2 & 8.7 \\
\hline \multirow[t]{3}{*}{ EFV } & 80 & 89.7 & 6.6 \\
\hline & 640 & 87.1 & 7.9 \\
\hline & 5120 & 93.3 & 8.7 \\
\hline \multirow[t]{3}{*}{ LPV } & 80 & 79.7 & 10.9 \\
\hline & 800 & 76.1 & 7.5 \\
\hline & 8000 & 82.2 & 7.3 \\
\hline \multirow[t]{3}{*}{ RTV } & 15 & 87.5 & 9.0 \\
\hline & 150 & 75.7 & 7.0 \\
\hline & 1500 & 81.6 & 6.1 \\
\hline
\end{tabular}

Table 4: Absolute Recovery of the determination of AZT, 3TC, d4T, EFV, NVP, LPV and RTV in human plasma $(n=5)$.

recovery determination. The data for absolute recovery are given in Table 4. The absolute recovery for 3TC and d4T were about $57 \%$ and $70 \%$, respectively, which were lower than that from the previous report [8]. It may be due to the complicated but cheap sample extraction procession. The recovery of other five analytes was high to $80 \%$ recovery, which indicated that our method was very suitable for analyzing these five analytes. For $\mathrm{d} 4 \mathrm{~T}$ and 3TC, although the lower recovery was detected, our research was not affected. As shown in Table 3, lower LOQ was detected in this work than the report [8] and 133 clinical samples were successfully analyzed.

Stock solution of all seven analysts and IS were stable at room temperature for $24 \mathrm{~h}$ and at $2-8^{\circ} \mathrm{C}$ for 30 days. 3TC, d4T, AZT, NVP, EFV, LPV and RTV in control human plasma at room temperature were stable at least for $56 \mathrm{~h}$ and for minimum of three freeze and thaw cycles. Spiked plasma samples stored at -20 C were stable for minimum 30 days. There was no significant degradation observed since the deviations in concentration was within $15 \%$ of their nominal values (Data not shown).

\section{Linearity}

The correlation coefficients $(r)$ of the calibration curves were $>0.99$ for all seven analytes as determined by linear analysis with a $1 / \mathrm{x}^{2}$ regression over a concentration range of $20-3200 \mu \mathrm{g} / \mathrm{L}$ for AZT, 3TC and $\mathrm{d} 4 \mathrm{~T}, 40-6400 \mu \mathrm{g} / \mathrm{L}$ for EFV and NVP, $62.5-10000 \mu \mathrm{g} / \mathrm{L}$ for LPV and $12.5-2000 \mu \mathrm{g} / \mathrm{L}$ for RTV respectively. The results showed that the linearity range of this method is wide and suit for clinical research in China.

\section{Accuracy and precision}

Intra-day and inter-day accuracy and precision was evaluated at three different concentrations (LQC, MQC and HQC) for each analyte. For inter-batch, three runs and for intra-batch, a single run was assayed. Each run consisted of five replicates.

The results of the precision and accuracy experiments were given in Table 5. Intra-day accuracy varied from $92.1 \%$ to $113.8 \%$ and interday accuracy from $95.3 \%$ to $107.4 \%$, respectively. While intra-day precision varied from $2.1 \%$ to $11.0 \%$ and inter-day from $6.1 \%$ to $10.9 \%$. These values are well within the acceptance criteria recommended by the China FDA guideline (2005, China Pharmacopoeia).

\begin{tabular}{|c|c|c|c|c|c|c|c|}
\hline \multirow[b]{2}{*}{ Analytes } & \multirow{2}{*}{$\begin{array}{l}\text { Concentration } \\
\left(\mu \mathrm{g} \cdot \mathrm{L}^{-1}\right)\end{array}$} & \multicolumn{3}{|c|}{ Intra-day $(\mathrm{n}=5)$} & \multicolumn{3}{|c|}{ Inter-day (n=15) } \\
\hline & & Mean & $\begin{array}{l}\text { Accuracy } \\
(\%)\end{array}$ & $\begin{array}{l}\text { Precision } \\
(\%)\end{array}$ & Mean & $\begin{array}{l}\text { Accuracy } \\
(\%)\end{array}$ & $\begin{array}{l}\text { Precision } \\
(\%)\end{array}$ \\
\hline \multirow[t]{3}{*}{ AZT } & 40 & 38.1 & 95.2 & 11.0 & 38.5 & 96.3 & 9.4 \\
\hline & 320 & 310.4 & 96.9 & 7.5 & 313.9 & 98.1 & 9.0 \\
\hline & 2560 & 2568.0 & 100.3 & 2.1 & 2610.7 & 102.0 & 6.8 \\
\hline \multirow[t]{3}{*}{ 3TC } & 40 & 40.2 & 100.6 & 8.7 & 38.1 & 95.3 & 8.5 \\
\hline & 320 & 297.6 & 93.0 & 4.6 & 308.5 & 96.4 & 8.5 \\
\hline & 2560 & 2516.0 & 98.2 & 10.4 & 2566.0 & 100.2 & 9.1 \\
\hline \multirow[t]{3}{*}{$\mathrm{d} 4 \mathrm{~T}$} & 40 & 37.6 & 94.0 & 10.1 & 38.2 & 95.4 & 8.7 \\
\hline & 320 & 302.0 & 94.4 & 4.8 & 312.3 & 97.6 & 9.4 \\
\hline & 2560 & 2586.0 & 100.9 & 2.1 & 2590.0 & 101.2 & 6.6 \\
\hline \multirow[t]{3}{*}{ NVP } & 80 & 79.5 & 99.4 & 5.9 & 80.1 & 100.2 & 7.6 \\
\hline & 640 & 662.8 & 103.5 & 9.0 & 658.3 & 102.9 & 7.0 \\
\hline & 5120 & 5044.0 & 98.5 & 6.1 & 4981.3 & 97.3 & 8.1 \\
\hline \multirow[t]{3}{*}{ EFV } & 80 & 73.7 & 92.1 & 7.1 & 77.4 & 96.7 & 9.2 \\
\hline & 640 & 651.8 & 102.0 & 7.7 & 648.7 & 101.4 & 8.4 \\
\hline & 5120 & 5178.0 & 101.1 & 5.1 & 5190.0 & 101.4 & 6.9 \\
\hline \multirow[t]{3}{*}{ LPV } & 80 & 91.1 & 113.8 & 6.6 & 85.4 & 106.7 & 10.9 \\
\hline & 800 & 885.0 & 110.8 & 3.6 & 844.7 & 105.7 & 7.5 \\
\hline & 8000 & 7900 & 98.7 & 3.8 & 7767.3 & 97.1 & 7.3 \\
\hline \multirow[t]{3}{*}{ RTV } & 15 & 14.9 & 99.1 & 9.6 & 15.0 & 99.7 & 9.0 \\
\hline & 150 & 152.6 & 101.9 & 9.0 & 161.1 & 107.4 & 7.0 \\
\hline & 1500 & 1494.0 & 99.8 & 4.9 & 1592.7 & 106.3 & 6.1 \\
\hline
\end{tabular}

Table 5: Precision and accuracy of AZT, 3TC, d4T, EFV, NVP, LPV and RTV in plasma $(n=5)$

\begin{tabular}{|l|l|l|l|}
\hline Antiretroviral agents & Samples $(\mathrm{n})$ & Mean \pm SD $(\mu \mathrm{g} / \mathrm{L})$ & Range $(\mu \mathrm{g} / \mathrm{L})$ \\
\hline AZT & 96 & $226.1 \pm 412.0$ & $5.11-2600$ \\
\hline 3TC & 133 & $1020 \pm 1412.1$ & $7.16-6020$ \\
\hline D4T & 35 & $199.6 \pm 262.7$ & $6.13-1030$ \\
\hline NVP & 52 & $5192.5 \pm 2264.6$ & $1880-12220$ \\
\hline EFV & 69 & $2429.06 \pm 1090.44$ & $875-5850$ \\
\hline LPV & 14 & $6529 \pm 573.2$ & $1320-20400$ \\
\hline RTV & 14 & $454.5 \pm 61.66$ & $62-2460$ \\
\hline
\end{tabular}

Table 6: The antiretroviral drug concentrations in Chinese patients. 


\section{Application of the method in clinical samples}

The validated method was successfully applied to detect the antiretroviral drug concentrations in Chinese patients with HIV infection. The observed plasma concentrations $(\mu \mathrm{g} / \mathrm{L})$ of AZT, 3TC, d4T, NVP, EFV, LPV and RTV are listed in Table 6. The observed antiHIV medicines plasma concentration and those postdosing time are presented in Figure 2. As shown in Figure 2, for 3TC, d4T and AZT, a large inter-patient variability of the drug concentrations $(\mu \mathrm{g} / \mathrm{L})$ was noted and our results were consistent with the previous report [13]. For NVP, there were 36 patients enrolled in this study, of which, 10 with drug concentrations higher than $6000 \mu \mathrm{g} / \mathrm{L}$ and 4 with drug concentration lower than $3400 \mu \mathrm{g} / \mathrm{L}$. According to a previous paper [14], the therapeutic range for NVP was $3400-6000 \mu \mathrm{g} / \mathrm{L}[15]$. So near $40 \%$ samples have the concentration beyond therapeutic range. For $\mathrm{EFV}$, there were 45 patients enrolled in this trial and plasma samples were collected from each subject, 1-4 samples per patient with a total of 69. There were 6 patients with the EFV concentrations beyond the therapeutic window $(1000-4000 \mu \mathrm{g} / \mathrm{L})$, one below $1000 \mu \mathrm{g} / \mathrm{L}$ and 5 above $4000 \mu \mathrm{g} / \mathrm{L}$. In this work, 14 samples containing LPV and RTV were analyzed. Our results showed a large inter-patient variability,with drug range $(\mu \mathrm{g} / \mathrm{L})$ from $1320-20400$ for LPV and 622460 for RTV. In the future, it will be necessary to use more samples and obtain more significant statistic data which can truly represent Chinese group.

\section{Discussion}

\section{LC-MS method}

To screen and quantify the currently available antiretroviral agents in China, a novel analytical method with high-throughout and lowcost is necessary. There are several HPLC and LC-MS/MS methods for measuring antiretroviral agents[8-11,16,17]. However, some of them [8-11] used SPE columns which is expensive, and some determined only two or three drugs in a combination therapy $[8,16,17]$ or certain classes of antiretrovirals $[18,19]$, which had lower throughout. So far a few methods have been previously reported for simultaneously determining NRTI, NNRTI and PI agents[10,20,21], however, in all
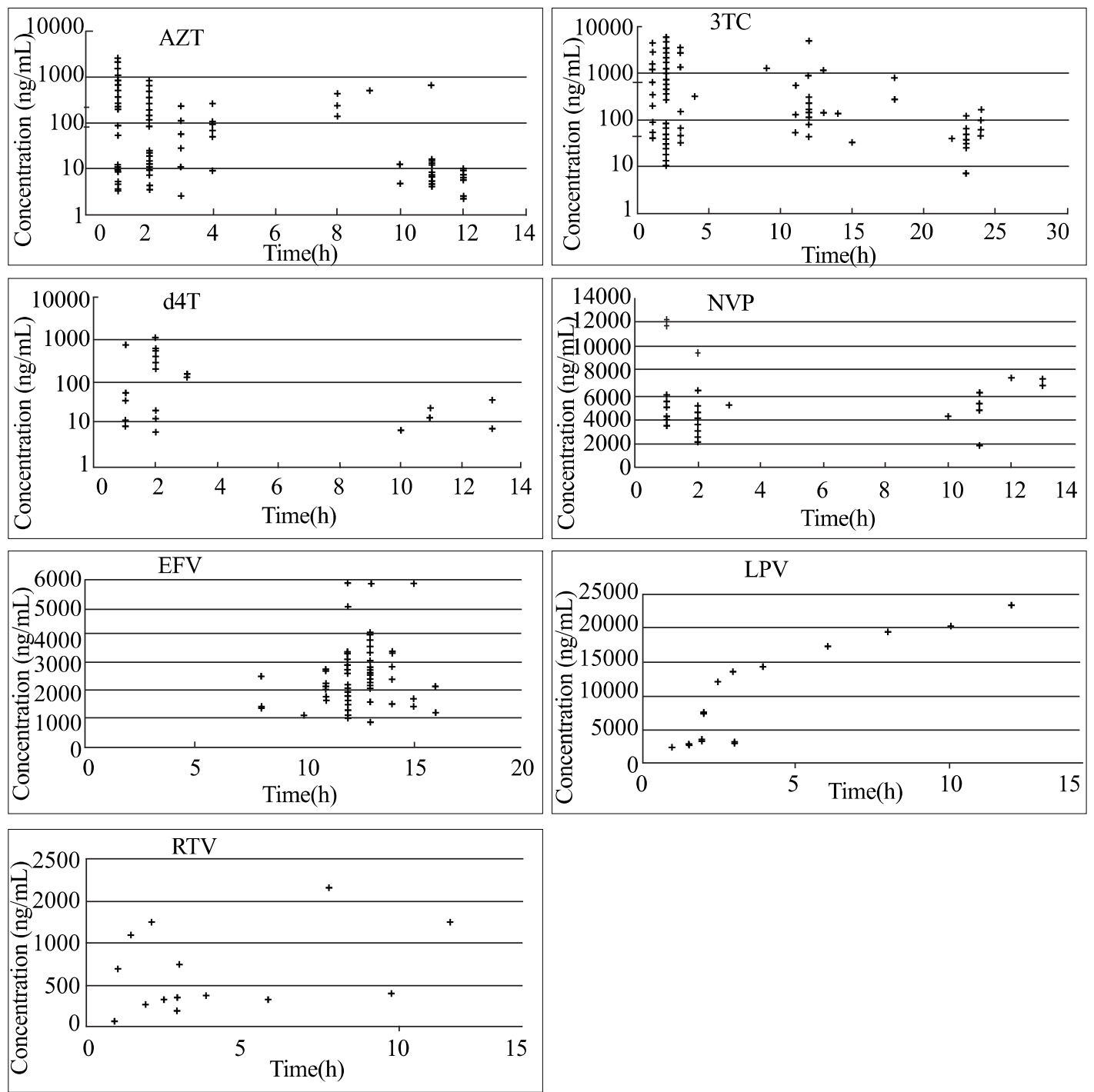

Figure 2: The plasma concentration of AZT, 3TC, d4T, NVP , EFV, LPV and RTV. 
these methods, both negative and positive MS/MS modes were used. This polarity switch might affect the replication and resolution of analysis method.

\section{Application of the method in clinical samples}

As described in introduction section, only few antivirus medicines are available in China and the dosage of these medicines is primarily based on the clinical data from Caucasians. So it is very necessary to carry out clinical research such as TDM, PK-PD, drug-drug interaction in China. In this study, 133 samples were analyzed and the concentration ranges of 7 anti-HIV drugs were shown in Table 6. These results show that the developed LC-MS method is an ideal method to measure drug concentrations in Chinese. However, due to the small sample size, the statistic data is not very significant and might not represent the whole Chinese population. In the future, it will be necessary to extend sample size and further study the pharmacokinetics of these anti-HIV drugs in the Chinese population and know the differences between other races and Chinese.

\section{Conclusion}

In conclusion, we developed and verified an LC-MS method for simultaneously quantifying the AZT, 3TC, d4T, EFV, NVP, LPV and RTV in plasma from Chinese patients infected by HIV. The sample preparation is cheap and easy for only protein precipitation followed by a liquid-liquid extraction. The LC-MS running time is short to 13 minutes. The main ARV medicines taken by Chinese were analyzed simultaneously. This method has lower LOQ [8] and wider quantification range [10] and is very suitable for PK-PD analysis, patient's adhesion detection and drug-drug interaction in China according the results from 133 clinical samples. To our knowledge, this was the first time to simultaneously quantify AZT, 3TC, d4T, EFV, NVP, LPV and RTV in Chinese patients with HIV infection and offer a right method for PK-PD analysis, drug-drug interaction and so on.

\section{Acknowledgment}

This work was supported by a Basic Research grant from the Ministry of Health, the People's Republic of China (WA-2007-04) and "National Key Technology R\&D Program of China (Grant No. 2008ZX10001-008)". We thank Professor Qing Ma from University at Buffalo for reviewing this manuscript. We also acknowledge the patients and the staff at Shanghai Public Health Clinical Center.

\section{References}

1. De Clercq E (2002) New developments in anti-HIV chemotherapy. Biochim Biophys Acta 1587: 258-275.

2. Gerber JG, Acosta EP (2003) Therapeutic drug monitoring in the treatment of HIVinfection. J Clin Virol 27: 117-128.

3. Kiser JJ, Anderson PL, Gerber JG (2005) Therapeutic drug monitoring: pharmacologic considerations for antiretroviral drugs. Curr HIVIAIDS Rep 2: 6167.

4. Durand-Gasselin L, Pruvost A, Dehée A, Vaudre G, Tabone MD, et al. (2008) High levels of zidovudine (AZT) and its intracellular phosphate metabolites in AZT- and AZT-lamivudine-treated newborns of human immunodeficiency virus-infected mothers. Antimicrob Agents Chemother 52: 2555-2563.

5. Holland DT, DiFrancesco R, Connor JD, Morse GD (2006) Quality assurance program for pharmacokinetic assay of antiretrovirals: ACTG proficiency testing for pediatric and adult pharmacology support laboratories, 2003 to 2004: a requirement for therapeutic drug monitoring. Ther Drug Monit 28: 367-374.
6. Back D, Gatti G, Fletcher C, Garaffo R, Haubrich R, et al. (2002) Therapeutic drug monitoring in HIV infection: current status and future directions. Aids 16: S5-37.

7. Poirier JM, Robidou P, Jaillon P (2005) Simple and simultaneous determination of the hiv-protease inhibitors amprenavir, atazanavir, indinavir, lopinavir, nelfinavir, ritonavir and saquinavir plus M8 nelfinavir metabolite and the nonnucleoside reverse transcriptase inhibitors efavirenz and nevirapine in human plasma by reversed-phase liquid chromatography. Ther Drug Monit 27: 186-192.

8. Mistri HN, Jangid AG, Pudage A, Gomes N, Sanyal M, et al. (2007) High throughput LC-MS/MS method for simultaneous quantification of lamivudine, stavudine and nevirapine in human plasma. J Chromatogr B Analyt Technol Biomed Life Sci 853: 320-332.

9. Rouzes A, Berthoin K, Xuereb F, Djabarouti S, Pellegrin I, et al. (2004) Simultaneous determination of the antiretroviral agents: amprenavir, lopinavir ritonavir, saquinavir and efavirenz in human peripheral blood mononuclear cells by high-performance liquid chromatography-mass spectrometry. J Chromatogr B Analyt Technol Biomed Life Sci 813: 209-216.

10. Jung BH, Rezk NL, Bridges AS, Corbett AH, Kashuba AD (2007) Simultaneous determination of 17 antiretroviral drugs in human plasma for quantitative analysis with liquid chromatography-tandem mass spectrometry. Biomed Chromatogr 21 : 1095-1104.

11. Fan B, Bartlett MG, Stewart JT (2002) Determination of lamivudine/stavudine/ efavirenz in human serum using liquid chromatography/electrospray tandem mass spectrometry with ionization polarity switch. Biomed Chromatogr 16: 383389 .

12. Matuszewski BK, Constanzer ML, Chavez-Eng CM (1998) Matrix effect in quantitative LC/MS/MS analyses of biological fluids: a method for determination of finasteride in human plasma at picogram per milliliter concentrations. Anal Chem 70: 882-889

13. Molto J, Blanco A, Miranda C, Miranda J, Puig J, et al. (2006) Variability in nonnucleoside reverse transcriptase and protease inhibitor concentrations among HIV-infected adults in routine clinical practice. Br J Clin Pharmacol 62: 560-566.

14. Smith PF, DiCenzo R, Morse GD (2001) Clinical pharmacokinetics of nonnucleoside reverse transcriptase inhibitors. Clin Pharmacokinet 40: 893-905.

15. Duong M, Buisson M, Peytavin G, Kohli E, Piroth L, et al. (2005) Low trough plasma concentrations of nevirapine associated with virologic rebounds in HIVinfected patients who switched from protease inhibitors. Ann Pharmacother 39 603-609.

16. Delahunty T, Bushman L, Robbins B, Fletcher CV (2009) The simultaneous assay of tenofovir and emtricitabine in plasma using LC/MS/MS and isotopically labeled internal standards. J Chromatogr B Analyt Technol Biomed Life Sci 877: 19071914

17. Huang Y, Gandhi M, Greenblatt RM, Gee W, Lin ET, et al. (2008) Sensitive analysis of anti-HIV drugs, efavirenz, lopinavir and ritonavir, in human hair by liquid chromatography coupled with tandem mass spectrometry. Rapid Commun Mass Spectrom 22: 3401-3409.

18. Rezk NL, Crutchley RD, Yeh RF, Kashuba AD (2006) Full validation of an analytical method for the HIV-protease inhibitor atazanavir in combination with 8 other antiretroviral agents and its applicability to therapeutic drug monitoring. The Drug Monit 28: 517-525

19. Rezk NL, Tidwell RR, KashubaAD (2004) High-performance liquid chromatography assay for the quantification of HIV protease inhibitors and non-nucleoside reverse transcriptase inhibitors in human plasma. J Chromatogr B Analyt Technol Biomed Life Sci 805: 241-247.

20. Volosov A, Alexander C, Ting L, Soldin SJ (2002) Simple rapid method for quantification of antiretrovirals by liquid chromatography-tandem massspectrometry. Clin Biochem 35: 99-103.

21. Ghoshal AK, Soldin SJ (2003) Improved method for concurrent quantification of antiretrovirals by liquid chromatography-tandem mass spectrometry. Ther Drug Monit 25: 541-543. 